\title{
Quality assessment of goji fruits, cranberries, and raisins using selected markers
}

\author{
Magdalena Jeszka-Skowron ${ }^{1} \cdot$ Karolina Oszust $^{2} \cdot$ Agnieszka Zgoła-Grześkowiak $^{1} \cdot$ Magdalena Frąc $^{2}$
}

Received: 31 May 2018 / Revised: 5 July 2018 / Accepted: 6 July 2018 / Published online: 1 August 2018

(c) The Author(s) 2018

\begin{abstract}
The aim of the study was to determine the antioxidant activity, total phenolic content, and total carotenoid content of dried fruit extracts prepared from goji fruits, cranberries, and raisins. Ergosterol content of samples-as a marker of fungal contamination-was measured by LC-MS/MS technique. For the goji fruit samples, fungal community-level physiological profiling and fungal isolate catabolic diversity were tested to evaluate more precisely their fungal contamination. The highest antioxidant activity and total phenolic content were determined for dried goji fruits. Simultaneously, the dried goji fruits were contaminated by ergosterol $-3.8 \mu \mathrm{g} \mathrm{g}{ }^{-1}$ of dried fruit and the bulk goji fruits were contaminated by heat-resistant fungi. The study of fungal community as a fungal contamination marker of dried goji fruits was performed by a combination of the Biolog FF plates and traditional culture methods which is the first application of this composite analytical approach. Ergosterol content could be an indicator of fungal growth on dried fruits. The Biolog FF microplates can be used as a quick, easy, cheap, and effective method for the detection of heat-resistant fungi in dried fruits.
\end{abstract}

Keywords Community-level physiological profiling $\cdot$ Heat-resistant fungi $\cdot$ Antioxidant activity $\cdot$ Dried fruits $\cdot$ Ergosterol

\section{Introduction}

Dried fruits such as raisins, cranberries, or goji fruits are becoming very popular, nowadays. They are usually added to cereals, teas, or other healthy foodstuffs. Dried fruits contain less carbohydrate than fresh foods, including lower level of glucose and fructose. They consist of fiber as well, but also contain such phytochemicals as phenolic acids, flavonoids, phytoestrogens, and carotenoids and such elements as copper, manganese, and germanium [1,2]. These compounds are in concentrated form compared to fresh fruits and their quantity depends on drying process. The appropriate quantity of dried fruits consumption has positive influence on glycaemic and insulin response in comparison to the fresh fruits [3]. One of the most popular dried fruits is raisins. Their regular consumption may reduce glycaemia and

Magdalena Jeszka-Skowron

magdalena.jeszka-skowron@ put.poznan.pl

1 Institute of Chemistry and Technical Electrochemistry, Poznan University of Technology, Berdychowo 4, 60-965 Poznan, Poland

2 Institute of Agrophysics, Polish Academy of Sciences, Doświadczalna 4, 20-290 Lublin, Poland cardiovascular risk factors, including blood pressure rate [4]. Phenolic acids such as caftaric and coumaric acids are distinctive for these fruits. Though loss of these compounds during the drying process reaches up to $90 \%$, raisins still possess high antioxidant activity [5].

The other rich sources of antioxidant are dried cranberries and goji fruits. Antioxidant activity of dried cranberries measured by DPPH (2,2-diphenyl-1-picrylhydrazyl) assay was higher than fresh kiwifruit, papaya, or pear [6]. The freeze-dried cranberry powder may improve serum lipids, and may show antioxidative effect by its protective function against protein and lipid oxidation [7]. Oszmiański et al. [8] determined $10.73 \mathrm{~g}$ of phenolic compounds in $100 \mathrm{~g}$ dried cranberries where peonidin 3-O-galactoside was the dominating flavonoid.

The quality of food on the market depends not only on the content of healthy compounds such as phenolics, but also on its microbiological contamination, e.g., by fungi. Ergosterol can be an indicator of fungal growth, also in food products [9-11]. Nevertheless, the enumeration of total number of fungi as their occurrence ascertainment is also a recognized and an important method in microbiological state evaluation of food products [12]. Identification for phenetic differences of fungi based on nutrient (within carbon sources) profiling 
might be a useful tool for food microbial contamination circumscription [13]. The Biolog FF (filamentous fungi) is the rapid spectrophotometric method for metabolic potential assessment of fungi and yeast. Evaluation of sole carbon source utilization patterns based on Biolog FF Plates is widely applied to compare environmental samples as far as profiling of fungal communities catabolic potentials or collating of fungal isolates catabolic activity [14-16].

Regarding the whole microbial population, the community-level physiological profiling (CLPPs) approach is admitted. Notwithstanding, for particular pure strain comparison, the fungal isolate catabolic diversity approach could be an appropriate corresponding appellative, since a set of isolate catabolic features are involved in the utilization of carbon sources located on the Biolog Plate. The CLPPs were originally designed based on ECO Plates and previously were applied mostly for soil samples [17, 18]. However, according to our best knowledge, there is a lack of scientific reports strictly concerning the evaluation of dried fruits fungal communities.

Drying is one of the most important processes in food industry. Removal of water from food is dictated by various advantageous effects of drying. First of all, it is connected with the lowering of water activity and moderation of many chemical enzymatic reactions and the practical elimination of microbial growth leading to stabilization of products. During goji fruits drying, attention should be paid to temperature of the process that allows to keep their rich nutritional properties. However, with no use of other sterilization, application of low temperature drying methods results in survival of heat-resistant fungi. Heat-resistant fungi can be defined as those capable of surviving temperatures at or above $75^{\circ} \mathrm{C}$ for 30 or more minutes [19]. The fungal structures, which can endure these temperatures, are ascospores, and, sometimes, chlamydospores, thick-walled hyphae or sclerotia [20,21]. Spoilage incidents involving heat-resistant fungi occurred increasingly in various examined products such as pasteurized fruits, dairy products, and soft drinks as reported by Houbraken and Samson [19]. Paecilomyces variotii, Fusarium oxysporum, Byssochlamys fulva, B. nivea, Talaromyces trachyspermus, and Neosartorya species were often encountered in fruits subjected to high temperatures $\left(>60{ }^{\circ} \mathrm{C}\right.$ ) even pasteurized. Therefore, it seems important to focus much more attention in food research on the detection of heat-resistant fungi in dried fruits, such as goji. Although dried fruits are important for human health as nutritional equivalent of fresh fruits with unique combination of taste, aroma, nutrients, and phytochemicals, according to Chang et al. [1], more research should be carried out to determine the complete metabolic profile of dried fruits in relation to their antioxidant activities or other bioactivities.

Therefore, the objectives of the study were to: (1) measure the antioxidant activity, total phenolic content, and total carotenoids content of dried fruit extracts; (2) determine the ergosterol content, the total number of fungi, and heatresistant fungi in dried fruits; (3) analyze fungal growth using community-level physiological profiling for total and heat-resistant fungi and evaluate catabolic potential of fungal strains isolated from dried fruits as a marker of fungal contamination.

\section{Materials and methods}

\section{Chemicals}

2,2-Diphenyl-1-picrylhydrazyl, gallic acid, ergosterol, and Folin-Ciocalteu's reagent were purchased from SigmaAldrich Chemical Co. (Steinheim, Germany). MS-grade acetonitrile and methanol were from POCH (Gliwice, Poland), and MS-grade formic acid was from Sigma-Aldrich. All other chemicals were of analytical grade.

High-purity water-deionized (DEMIWA 5 ROSA, Watek, Czech Republic) and doubly distilled (quartz apparatus, Bi18, Heraeus, Hanau, Germany)—was used throughout the research. The resistivity of the water was $18 \mathrm{M} \Omega \mathrm{cm}$.

\section{Material}

Dried fruits including three goji fruits: Bulk-China (C1), Package-BIO-China (C2) and Package-China-Tibet (C3); 2 cranberries: Bulk-USA and Package-USA; 2 raisins: Package-King'-Turkey and Package-Sultana-Turkey were purchased from a local market. These samples are the most popular dried fruits sold in Poland. The abbreviation BIO given for the $\mathrm{C} 2$ sample means that the product comes from organic farming.

\section{Extraction processes}

After cutting and homogenization of samples in the mill (IKA A11 basic, Ika Poland), $1 \mathrm{~g}$ of fruits was extracted by $25 \mathrm{~mL}$ of ethanol with distilled water $(1: 1 ; \mathrm{v} / \mathrm{v})$ to determine antioxidant activity and ethanol with acetone $(1: 1 ; \mathrm{v} / \mathrm{v})$ to determine carotenoid content for 30 min with ultrasoundassisted extraction. The obtained extract was decanted, and filtered through $0.45 \mu \mathrm{m}$ polytetrafluoroethylene syringe filter from Agilent Technologies (Santa Clara, CA, USA), and finally diluted to proper volume with distilled water. The fruit extract was prepared directly before analysis.

\section{Total phenolic content}

Total phenolic content of dried fruit extracts was determined using Folin-Ciocalteu's reagent [22]. Gallic acid was used as an external standard (the linearity $r^{2}=0.999$ 
and calibration curve range: $5-100 \mu \mathrm{g} \mathrm{mL} \mathrm{m}^{-1}$ of gallic acid solutions $y=0.0089 \mathrm{x}-0.0105$ ). The absorbance of samples against a reagent blank was measured at $754 \mathrm{~nm}$ after $1 \mathrm{~h}$ of incubation at room temperature protected from light. The results were expressed as gallic acid equivalent (GAE) in $\mathrm{mg}$ $\mathrm{g}^{-1}$ of the extract. The other parameters of validation were limits of detection and quantification: $\mathrm{LOD}=3.89 \mu \mathrm{g} \mathrm{mL}^{-1}$ and $\mathrm{LOQ}=11.12 \mu \mathrm{g} \mathrm{mL}^{-1}$. These values were estimated as $3.3 \times$ standard deviation of 6 blanks/the slope of the calibration curve.

\section{Total carotenoid content}

The determination of total carotenoids content was conducted according to the procedure described previously [23]. Zeaxantin $(\lambda=452 \mathrm{~nm})$ was chosen as a dominating carotenoid in goji fruits. The total carotenoids in the acetone-ethanol extracts were determined as zeaxantin at $452 \mathrm{~nm}$ [24] and the values were defined as in Eq. 1, using the specific absorption coefficient in acetone:

Total carotenoids $\left(\mathrm{mg} \mathrm{g}^{-1}\right)=A \times V \times 10^{3} / A_{1 \mathrm{~cm}} \times 100 \times m_{\mathrm{s}}$,

where $A$-absorbance of sample, $V$-volume of sample $(\mathrm{mL}), m_{\mathrm{s}}$-dry mass of sample $(\mathrm{g})$, and $A_{1 \mathrm{~cm}}$-specific absorptivity.

\section{Antioxidant activity}

The ability of dried fruit extracts to scavenge DPPH radicals (antioxidant activity) was studied according to the method Jeszka-Skowron and Zgoła-Grześkowiak [25]. Briefly, dried fruit extract was diluted in methanol $(1: 10 ; \mathrm{v} / \mathrm{v})$, and then, $1.0 \mathrm{~mL}$ of $0.5 \mathrm{mmol} \mathrm{L}^{-1}$ methanol solution of DPPH was mixed with $3 \mathrm{~mL}$ of the extract. Next, the solution was mixed and left for $30 \mathrm{~min}$ at room temperature protected from light. The absorbance of samples against a reagent blank was measured at $516 \mathrm{~nm}$. Antioxidant activity was expressed as percentage DPPH scavenging relative to control solution using the following equation:

$$
\begin{aligned}
\text { Antioxidant activity }(\%)= & ((\text { Absorbance of control } \\
& - \text { Absorbance of sample }) / \\
& (\text { Absorbance of control })) \\
& \times 100 \% .
\end{aligned}
$$

The method performance was validated in terms of linearity (correlation coefficient $-r^{2}=0.9993$ ) where Trolox solutions $\left(20-140 \mu \mathrm{mol} \mathrm{L}^{-1}\right)$ were used as a standard for the calibration curve $(y=0.6879 x-1.890)$. The limits of detection and quantification were $\mathrm{LOD}=0.021 \mu \mathrm{g} \mathrm{mL} \mathrm{L}^{-1}$ and $\mathrm{LOQ}=0.065 \mu \mathrm{g} \mathrm{mL}^{-1}$.
All spectrophotometric determinations were performed with the use of Beckman UV-Vis Spectrophotometer $7500 \mathrm{DU}$ (Brea, CA, USA) with glass cuvettes of $1 \mathrm{~cm}$ optical length. Spectra were recorded in the range from 380 to $800 \mathrm{~nm}$ with $0.2 \mathrm{~nm}$ resolution. All determinations were carried out in triplicate. The precision (RSD) estimated as $\%$ of standard deviation to the average of the result in all spectrophotometric determinations was below 3.0\%

\section{Ergosterol content using liquid chromatography- mass spectrometry}

The determination of ergosterol content was conducted according to the procedure described previously $[2,11]$. The UltiMate 3000 RSLC chromatographic system from Dionex (Sunnyvale, CA, USA) was used. $10 \mu \mathrm{L}$ samples were injected into a Gemini-NX C18 column $(100 \mathrm{~mm} \times 2.0 \mathrm{~mm}$ ID; $5 \mu \mathrm{m}$ ) from Phenomenex (Torrance, CA, USA) maintained at $35^{\circ} \mathrm{C}$. The isocratic mobile phase employed during the analysis consisted of methanol:water $(95: 5, \mathrm{v} / \mathrm{v})$ at a flow rate of $0.4 \mathrm{~mL} \mathrm{~min}^{-1}$. The LC column effluent was directed to the API 4000 QTRAP triple quadrupole mass spectrometer from AB Sciex (Foster City, CA, USA) using the atmospheric pressure chemical ionization (APCI) interface. The APCI source operated in positive-ion mode. The following settings for the ion source and mass spectrometer were used: curtain gas $10 \mathrm{psi}$, nebulizer gas $20 \mathrm{psi}$, temperature $400{ }^{\circ} \mathrm{C}$, nebulizing current $3 \mu \mathrm{A}$, and collision gas $10 \mathrm{psi}$. Declustering potential was $65 \mathrm{~V}$ and the dwell time was set to $200 \mathrm{~ms}$. The quantitative transition was from 379.3 to $69.1 \mathrm{~m} / \mathrm{z}$ at collision energy set to $45 \mathrm{~V}$ and the confirmatory transition was from 379.3 to $145.1 \mathrm{~m} / \mathrm{z}$ at collision energy set to $22 \mathrm{~V}$. The method has been validated during our previous studies. Validation included: precision, recovery, linearity, limits of detection and quantification, and matrix effect evaluation. On the basis of obtained results, the method has been found suitable for the determination of ergosterol in dried fruit samples [2].

\section{Fungal community-level physiological profiling of goji fruits}

Community-level physiological profiling was assessed using FF microplate system (Biolog Inc., Hayward, CA, USA). Each FF microplate consists of 95 different sole carbon sources and a water blank. FF microplates were used to determine the carbon source use patterns of heat-resistant fungal community of each dried goji fruit sample. The goji samples $(1 \mathrm{~g})$ were homogenized and shaken in $99 \mathrm{~mL}$ of saline peptone water (Biocorp, Warsaw, Poland) for $20 \mathrm{~min}$ before incubating for $30 \mathrm{~min}$ at $4{ }^{\circ} \mathrm{C}$. Thermal shock $\left(30 \mathrm{~min}\right.$ at $\left.80^{\circ} \mathrm{C}\right)$ was applied to provide the heat-resistance fungal community to develop. This step was followed by filtration, and $100 \mu \mathrm{L}$ of the sample 
suspension was inoculated into each well of FF microplate and incubated at $30{ }^{\circ} \mathrm{C}$. Goji fruits that were not subjected to the shock were used as controls. The carbon source utilization rate was recorded by reading of turbidity reflecting mycelia growth of fungal community at $750 \mathrm{~nm}$ using a microplate reader (Biolog $\left.{ }^{\mathrm{TM}}\right)$. The data were recorded in triplicate every $24 \mathrm{~h}$ for up to $240 \mathrm{~h}$. Optical density higher than 0.25 for each substrate was considered as a positive response of the particular strain. The microbial catabolic potential of goji fruits fungal community was expressed as the average well density development (AWDD) and the substrate richness index was calculated to evaluate the number of substrates utilized by each strain during incubation time. In addition, the percentages of the carbon sources that were used by groups were evaluated. As far as AWDD index corresponds to average well color development index (AWCD), it is widely used for colorimetric changes connected to tetrazolium violet transformations, while substrate utilization is usually provided on data readings at $590 \mathrm{~nm}$. In this experiment, it was decided to perform calculations on optical density data (OD, $750 \mathrm{~nm}$ ), since a set of colored substances extracted from goji fruits within homogenization significantly increased colorimetric values (they were out of measurement range).

\section{Fungal enumeration in dried fruits}

The total number of fungi was determined using the plate method with Chloramphenicol LAB-AGAR (Biocorp, Warsaw, Poland) and the serial dilution method. Dried fruits $(10 \mathrm{~g})$ were homogenized with $90 \mathrm{~mL}$ of saline peptone water (Biocorp, Warsaw, Poland) and filtered. Aliquots of the suspensions were transferred to Petri dishes with the medium using pour plates. The plates were cultivated for 7 days at $27^{\circ} \mathrm{C}$ and counted.

\section{Isolation of heat-resistant fungi strains from goji fruits}

Heat-resistant fungi strains isolation was performed using the modified method of Houbraken and Samson [19]. $25 \mathrm{~g}$ of each dried goji fruits sample were homogenized in $37.5 \mathrm{~mL}$ of sterile water. The thermal shock $\left(30 \mathrm{~min}\right.$ at $80^{\circ} \mathrm{C}$ in a water bath) was applied. Homogenized materials were mixed with $62.5 \mathrm{~mL}$ double strength Malt Extract Agar medium (Biocorp Polska, Warsaw, Poland) and antibiotics, and applied on five Petri dishes and incubated for 21 days at $30{ }^{\circ} \mathrm{C}$. Eight pure fungal strains (G28/15, G29/15, G31/15, G41/15, G42/15, G45/15, G65/15, and G66/15), supposed to be heat-resistant, were isolated and cultured on Potato Dextrose Lab-Agar (PDA) (Biocorp Polska) for further catabolic diversity comparison.

\section{Fungal isolate catabolic diversity}

Catabolic diversity of heat-resistant isolates was generated based on the growth intensity of the organism on 95 particular substrates, low-molecular-weight carbon sources, and assessed using the FF microplates (Biolog $\left.{ }^{\mathrm{TM}}\right)$. The inoculation procedure was based on the method according to manufacturer's protocol modified by Frąc [17]. To prepare inoculum, each fungal isolate was obtained by cultivation on potato dextrose agar in the dark at $30{ }^{\circ} \mathrm{C}$ during 10 days. After the suspension of the culture was homogenized in inoculating fluid (FF-IF, Biolog ${ }^{\mathrm{TM}}$ ) the transmittance was adjusted to $75 \%$ using a turbidimeter (Biolog ${ }^{\mathrm{TM}}$ ). $100 \mu \mathrm{l}$ of the above-mentioned suspension was added to each well and the inoculated microplates were incubated at $30{ }^{\circ} \mathrm{C}$ during 10 days. The optical density at $750 \mathrm{~nm}$ was determined using a microplate reader (Biolog ${ }^{\mathrm{TM}}$ ) every day, in triplicate. Catabolic diversity was determined by the number of different substrates utilized by the individual isolates, and expressed as substrate richness $(R)$ and AWDD indices.

\section{Statistical analysis}

The results of phenolics, total carotenoids, and antioxidant activity and ergosterol analyses were expressed as mean \pm standard deviation (at least three replications). Analysis of variance (ANOVA) and significant differences among means by Tukey's test were implemented. The significance level was based on a confidence level of $95 \%$. The experimental data were analyzed using Statistica 12.5 program (StatSoft Inc., Tulsa, OK, USA).

To determine groups in the data set, cluster analysis was applied to determine the utilization of carbon substrate by the particular fungal strain isolated from goji fruits. Groupings were followed according to the stringent Sneath criterion (33\%), and less restrictive criterion (66\%), respectively. After grouping, the ANOVA and Tukey's test were used which allowed to conclude about strains growth on individual carbon sources.

\section{Results}

\section{Total phenolic content, carotenoid content, and antioxidant activity of dried fruits}

The highest amount of total phenolic content was determined for Bulk-China goji fruits and all goji extracts contained three-fourfold higher quantity of these compounds than extracts from cranberries or raisins (Table 1). Carotenoid content of goji berries was significantly different between all the analyzed samples $(p \leq 0.05)$ and PackageBio-China goji fruits possessed almost threefold higher 
Table 1 Total phenolic content, carotenoid content, and antioxidant activity of dried fruits

\begin{tabular}{llll}
\hline Sample & $\begin{array}{l}\text { Total phenolic content } \\
\left(\mathrm{mg} \mathrm{GAE} \mathrm{g}^{-1}\right)\end{array}$ & Carotenoids $\left(\mathrm{mg} \mathrm{g}^{-1}\right)$ & Antioxidant activity (\%) \\
\hline Goji fruits & & & \\
$\quad$ Bulk-China & $27.02^{\mathrm{e}} \pm 0.38$ & $186.4^{\mathrm{a}} \pm 3.2$ & $81.38^{\mathrm{c}} \pm 0.09$ \\
Package-BIO-China & $24.22^{\mathrm{d}} \pm 0.25$ & $556.2^{\mathrm{c}} \pm 5.1$ & $83.76^{\mathrm{d}} \pm 0.43$ \\
Package-China-Tibet & $24.25^{\mathrm{d}} \pm 0.69$ & $462.3^{\mathrm{b}} \pm 5.6$ & $81.89^{\mathrm{c}} \pm 0.03$ \\
Cranberry fruits & & & \\
Bulk-USA & $7.97^{\mathrm{c}} \pm 0.57$ & nd & $38.40^{\mathrm{b}} \pm 1.19$ \\
Package-USA & $6.67^{\mathrm{b}} \pm 0.52$ & nd & $34.92^{\mathrm{a}} \pm 0.55$ \\
Raisins & & & $36.71^{\mathrm{a}} \pm 0.65$ \\
Package-Kings'-Turkey & $8.34^{\mathrm{c}} \pm 0.60$ & nd & $35.73^{\mathrm{a}} \pm 0.70$ \\
Package-Sultana-Turkey & $5.03^{\mathrm{a}} \pm 0.35$ & nd & \\
\hline
\end{tabular}

Averages in a column with different letters are significantly different (Tukey's test; $p \leq 0.05$ ) \pm SD (three repetitions)

$n d$ not detected (<LOD)
Table 2 Ergosterol content and total content of fungal growth/molds after 7 days of incubation

\begin{tabular}{lcl}
\hline Sample & Ergosterol $\left(\mathrm{ng} \mathrm{g}^{-1}\right)$ & $\begin{array}{l}\text { Total content } \\
\text { of fungi/molds } \\
(\mathrm{cfu})\end{array}$ \\
\hline Goji fruits & & \\
Bulk-China & $3781.4^{\mathrm{f}} \pm 90.6$ & $28 / 23$ \\
Package-BIO-China & $358.7^{\mathrm{d}} \pm 18.7$ & $0^{*}$ \\
Package-China-Tibet & $699.3^{\mathrm{e}} \pm 22.8$ & $0^{*}$ \\
Cranberry fruits & & \\
Bulk-USA & $34.6^{\mathrm{c}} \pm 2.0$ & $0^{*}$ \\
Package-USA & $27.2^{\mathrm{b}} \pm 3.3$ & $0^{*}$ \\
Raisins & & \\
Package-Kings'-Turkey & $15.1^{\mathrm{a}} \pm 1.9$ & $0^{*}$ \\
Package-Sultana-Turkey & $27.6^{\mathrm{b}} \pm 3.8$ & $0^{*}$ \\
\hline
\end{tabular}

Averages in a column with different letters are significantly different (Tukey's test; $p \leq 0.05) \pm \mathrm{SD}$ (three repetitions)

$c f u$ colony-forming units

*No colony was grown after 7 day incubation at $27^{\circ} \mathrm{C}$

carotenoids content in comparison to Bulk-China goji fruits. Carotenoids content and antioxidant activity of goji fruit extracts were the highest for Package-Bio-China goji fruits. Cranberry extracts and raisins extracts showed similar antioxidant activity in DPPH assay (with one exception) which was about twofold lower than that noted for goji extracts (Table 1).

\section{Ergosterol content and fungal growth}

Ergosterol was found in every tested sample (Table 2). Very high concentration of this marker was found in Bulk-China goji fruits and 5-10 times lower concentrations were found in other goji samples. Cranberries and raisins fruits also contain ergosterol, but its concentration was from 10- to 100 -fold lower than in goji samples.

Apart from ergosterol, total content of fungi/molds in goji, cranberries, and raisins was analyzed. After 7 days of incubation at $27{ }^{\circ} \mathrm{C}$, only Bulk-China goji fruit samples contained fungi/molds. There was no fungal growth observed in the other tested fruits, i.e., cranberries and raisins. Therefore, only goji fruits samples were taken to the subsequent analyses such as FF microplate analysis.

\section{Fungal community-level physiological profiling of goji fruits}

Cluster analysis performed on data set of goji fruits generated from FF microplates for fungal communities revealed differences in ability of particular communities to grow on carbon sources, as shown in Fig. 1. The most active community was found in the Package-China-Tibet goji fruits (C3), which was comparable to that found in the PackageBIO-China (C2), what can be noted at heat map in terms of amount of the most intensively utilized $\mathrm{C}$ substrates $\left(\mathrm{OD}_{750 \mathrm{~nm}}>0.5\right) . \mathrm{C} 3$ and $\mathrm{C} 2$ grouped together in one cluster (marked as square), when the Sneath criterion (66\%) was followed (Fig. 2a). Bulk-China goji fruit (C1) community revealed the weakest ability for $\mathrm{C}$-source utilization, among tested fruits, as reflected by not reaching the growth level $\mathrm{OD}_{750 \mathrm{~nm}}>0.5$. However, the significant number of substrates for $\mathrm{C} 1$ that were on the level when the utilization starts $\left(\mathrm{OD}_{750 \mathrm{~nm}}>0.5\right)$ was noted, as shown by AWDD (Fig. 3Aa) and Richness (Fig. 3Ab) indices. In spite of heat shock, Richness of $\mathrm{C} 1$ HS was not substantially different from $\mathrm{C} 3$ and $\mathrm{C} 2$ (where, there was no temperature stress endured). Strong influence of heat shock (HS samples) treatment could be observed for all tested goji fruits. 


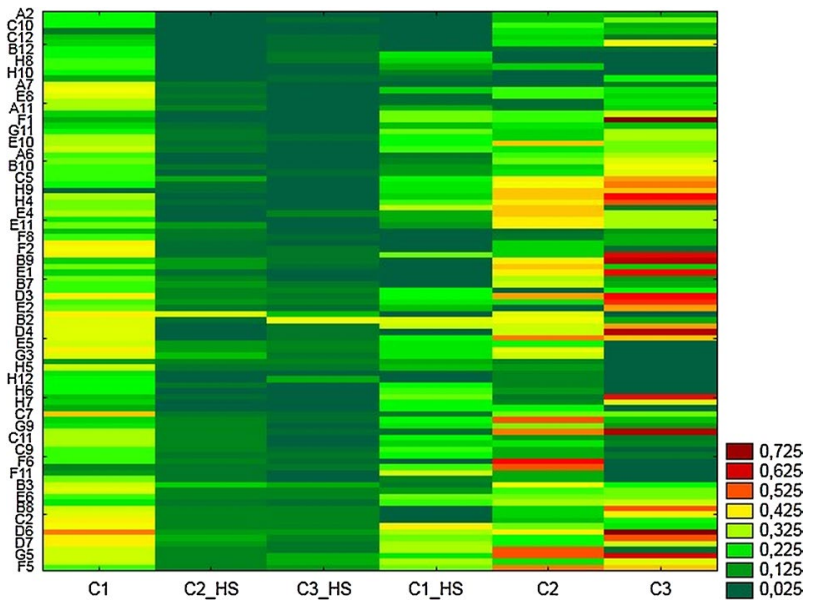

Fig. 1 Cluster analysis performed on data set of goji fruits (C1-C3) generated from $\mathrm{FF}$ microplates for fungal communities (98 different carbon sources). C1 Bulk-China goji fruit, C1-HS Bulk-China goji fruit after heat shock, C2 Package-BIO-China, C2-HS PackageBIO-China after heat shock, C3 Package-China-Tibet goji fruits, C3-HS Package-China-Tibet goji fruits after heat shock, A2 Tween 80, $A 3 \mathrm{~N}$-acetyl-D-galactosamine, $A 4 \mathrm{~N}$-acetyl-D-glucosamine, $A 5$ $N$-acetyl-D-mannosamine, $A 6$ adonitol, $A 7$ amygdalin, $A 8$ D-arabinose, $A 9$ L-arabinose, $A 10$ D-arabitol, $A 11$ arbutin, $A 12$ D-cellobiose, $B 1$ alfa-cyclodextrin, $B 2$ beta-cyclodextrin, $B 3$ dextrin, $B 4$ i-erythritol, $B 5$ D-fructose, $B 6$ L-fucose, $B 7$ D-galactose, $B 8$ D-galacturonic Acid, $B 9$ gentiobiose, $B 10$ D-gluconic acid, B11 D-glucosamine, $B 12$ alfa-D-glucose, $C 1$ glucose-1-phosphate, $C 2$ glucuronamide, $C 3$ D-glucuronic acid, C4 glycerol, C5 glycogen, C6 m-inositol, C7 2-keto-D-gluconic acid, $C 8$ alfa-D-lactose, $C 9$ lactulose, $C 10$ maltitol, $C 11$ maltose, $C 12$ maltotriose, D1 D-mannitol, D2 D-mannose, $D 3$ D-melezitose, D4 D-melibiose, D5 alfa-methyl-D-galactoside, D6 beta-methyl-D-galactoside, $D 7$ alfa-Methyl-D-glucoside, $D 8$ betamethyl-D-glucoside, $D 9$ palatinose, D10 D-psicose, D11 D-raffinose, $D 12$ L-rhamnose, E1 D-ribose, E2 salicin, E3 sedoheptulosan, E4 D-sorbitol, E5 L-sorbose, E6 stachyose, E7 sucrose, E8 D-tagatose, $E 9$ D-trehalose, E10 turanose, E11 xylitol, E12 D-xylose, F1 gammaamino-butyric acid, $F 2$ bromosuccinic acid, F3 fumaric acid, F4 beta-hydroxy-butyric acid, $F 5$ gamma-hydroxy-butyric acid, F6 p-hydroxyphenylacetic acid, $F 7$ alfa-keto-glutaric acid, $F 8$ D-lactic acid methyl ester, $F 9$ L-lactic acid, $F 10$ D-malic acid, $F 11$ L-malic acid, F12 quinic acid, G1 D-saccharic acid, G2 sebacic acid, G3 succinamic acid, $G 4$ succinic acid, $G 5$ succinic acid mono-methyl ester, $G 6 \mathrm{~N}$-acetly-L-glutamic acid, $G 7$ alaninamide, $G 8$ L-alanine, $G 9$ L-alanyl-glycine, $G 10$ L-asparagine, $G 11$ L-aspartic acid, $G 12$ L-glutamic acid, $H 1$ glycyl-L-glutamic acid, $H 2$ L-ornithine, $H 3$ L-phenylalanine, $H 4$ L-proline, $H 5$ L-pyroglutamic acid, $H 6$ L-serine, $H 7$ L-threonine, $H 8$ 2-amino ethanol, $H 9$ putrescine, $H 10$ adenosine, $H 11$ uridine, $H 12$ adenosine-5'-monophosphate

Fungal communities of Package-China-Tibet and PackageBIO-China after heat shock (C3_HS and C2_HS, respectively) lost their ability to utilize $\mathrm{C}$ substrates, and therefore, their growth was almost not observed. Although the level of the $\mathrm{C} 1$ community growth before heat shock did not reach $\mathrm{OD}_{750 \mathrm{~nm}}>0.5$, after treatment, the community (C1_HS) was able to metabolize most of the substrates on higher level than the other treated communities.
Figure 4 presents $\mathrm{C}$-substrate group utilization pattern of goji fruit fungal communities generated from FF microplates using Biolog ${ }^{\mathrm{TM}}$ System. There was great reduction met in percentage of amino acid group utilization after heat stress for all the tested goji fruits $[8,5$, and $15 \%$ for Bulk China (C1), Package-BIO-China (C2), and Package-China-Tibet (C3)]. For C1, it was related to simultaneous increase in carbohydrate and others group utilization (4 and 7\% upswing, respectively). Regarding $\mathrm{C} 2$, the greater accompanying utilization level was met for amines/amides (4\%), carboxylic acids $(6 \%)$, and decrease in polymers utilization (7\%). As for $\mathrm{C} 3$, the reduction of amino acids was linked to greater development of fungal community on amines/amides, carboxylic acids, polymers, and others group (noted increase was at level about $5,8,11$, and $5 \%$, respectively).

\section{Fungal isolate catabolic diversity}

It is worth noting that observed activity in C1_SH can be explained by the occurrence of heat-resistant fungi on Bulk-China goji fruit (C1), what was confirmed by heatresistant fungi strains isolation from $\mathrm{C} 1$ goji fruits. The isolates were named as follows: G28/15, G29/15, G31/15, G41/15, G42/15, G45/15, G65/15, and G66/15. The catabolic diversity comparison of particular isolates, expressed as (a) substrate richness (b) average well density development, was determined. Cluster analysis-based dendrogram, presented in Fig. 2b, shows correlation between heat-resistant fungal isolates in relation to utilization of $\mathrm{C}$ sources from the FF microplate. Irrespective of the Sneath criterion: stringent (33\%) or less restrictive criterion $(66 \%)$ (Fig. 2a), particularly isolates, represent completely different catabolic activity, as far as C-substrate utilization was considered. The isolates differed when AWDD was taken into consideration (Fig. 3Ba). The greatest AWDD value was noted for G45/15 isolate, and significantly lowest for G29/15 and G41/15. Regarding richness index, the best result was obtained for G45/15 isolate, whereas the others did not differ significantly. Furthermore, the AWDD and R values for isolates (Fig. 3Ba, Bb, respectively) were lower than for community samples (Fig. 3Ba, Bb respectively), which means that there were heat-resistant strains dwelling in community featured with greater capability to utilize C substrates (catabolic potential) located on FF plates comparing to pure isolates separately.

\section{Discussion}

The quality of food depends on the high level of bioactive compounds and its package condition. However, it was found that packaging in separate bags had no influence on antioxidant activity of dried fruits. It was shown that extracts 


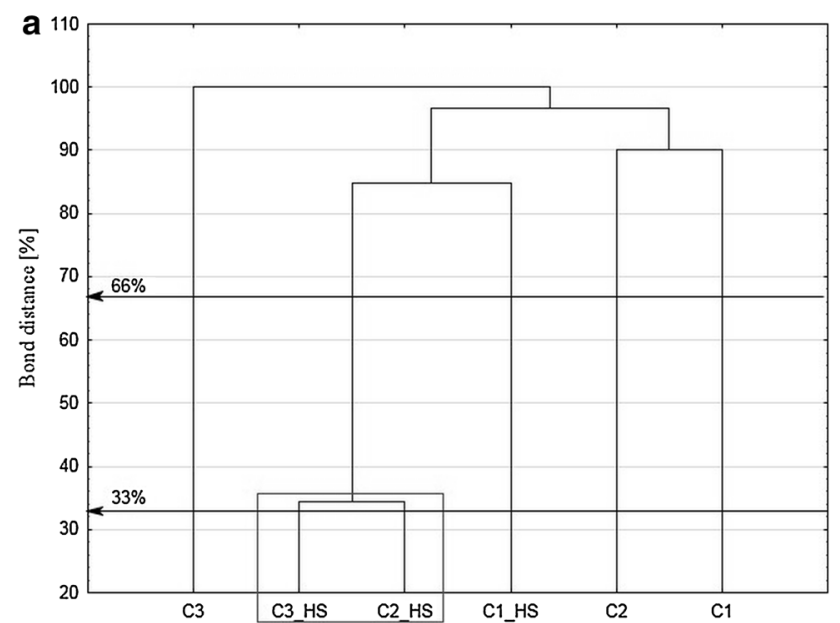

Fig. 2 Euclidian distance allowed the cluster-joining analysis for a dried goji samples and b strains of goji $\mathrm{C} 1$ sample. $C 1$ Bulk-China goji fruit, C1-HS Bulk-China goji fruit after heat shock, C2 Package-

prepared from goji fruits revealed higher antioxidant activity than the extracts of cranberries and raisins. Goji fruits contain carotenoids, and therefore, antioxidant activity of these fruits was higher than that of the other fruits [26].

Dried goji fruit water extracts were examined following Endes et al. [27] and these extracts contained $3.44 \pm 0.37 \mathrm{mg}$ GAE $100 \mathrm{~mL}^{-1}$ of total phenolics, and antioxidant activity was $20.78 \% \pm 1.29$ measured by DPPH assay. However, these results are difficult to compare with the results from this study due to different extraction solvent (water vs. ethanol-water) and slightly different preparation of assays.

Carotenoids content was similar with the other author findings in the range $0.03-0.5 \%$ of the dried goji fruit [26, 28]. Packed goji fruits contain higher amount of carotenoids than the bulk ones. Carotenoids are very sensitive compounds, and therefore, their concentration was decreased for goji without package [29].

The results of total phenolic content for extracts prepared from cranberries and raisins were not significantly different. These results are slightly different from the other authors' reports. Ishiwata et al. [6] measured total phenolic content of dried fruits and the highest phenolic content was determined for raisins. On the other hand, Vinson et al. [30] showed that phenolic contents in dried cranberry fruits $(870 \pm 277 \mathrm{mg}$ catechins of $100 \mathrm{~g}$ fresh weight) were higher than in raisins (551 $\pm 46 \mathrm{mg}$ catechins of $100 \mathrm{~g}$ fresh weight).

Apart from the influence of positive compounds on the quality of dried fruits, the negative aspects in food control should also be taken under consideration while assessing their quality. The potential microbial spoilage during storage and the potential health hazard due to fungal presence and mycotoxins accumulation is one of the most important concerns regarding to dried fruits [31, 32]. It has been shown

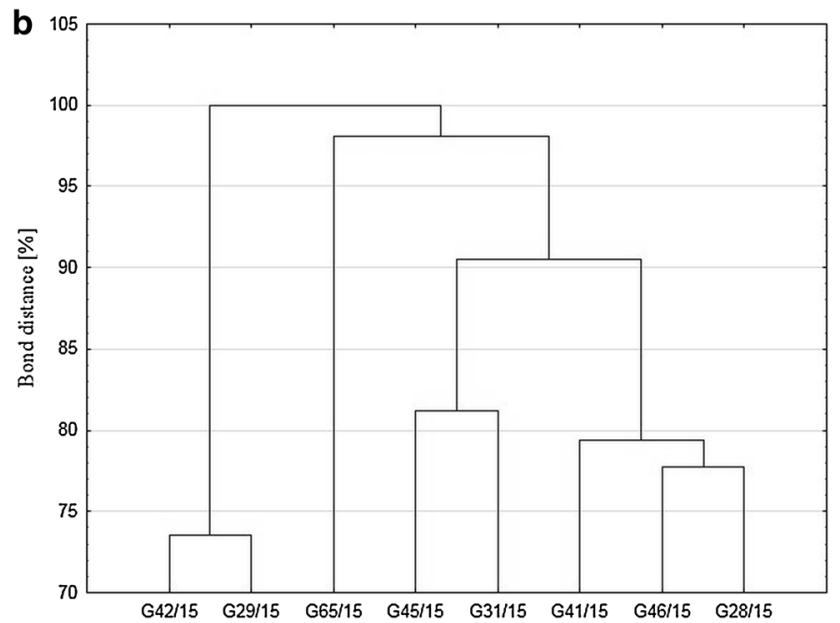

BIO-China, C2-HS Package-BIO-China after heat shock, C3 Package-China-Tibet goji fruits, C3-HS Package-China-Tibet goji fruits after heat shock

that dried fruits can be contaminated by fungi and their mycotoxins [33, 34], and therefore, monitoring is critical for such fruit quality. However, the phenotype microarray method has not previously been described as a tool for the fast detection of mycological contamination, especially for heat-resistant fungi in dried fruits. Recent studies [32-34] documented the occurrence of high level of mycotoxins or fungal cells in dried fruits including apricots, dates, prunes, figs, and raisins. In contrast to earlier findings, in this study, no evidence of fungi presence in dried raisins was detected. However, the findings of the current study indicated the presence of fungi in dried goji fruits, including heat-resistant fungi, especially yeast. Moreover, the results were confirmed by both the conventional culture and community-level physiological profile methods. These results were supported by high level of ergosterol in the sample from which fungal species were isolated. Thus, it was confirmed that high amount of ergosterol ( $3.8 \mu \mathrm{g} \mathrm{g}^{-1}$ of dried fruits) is an indicator of fungal growth. It is difficult to explain that, among three of studied goji fruit samples, only one was contaminated according to the conventional method. However, this result might be related to packing, transport, and storage conditions. It is known that ergosterol found in the samples can be extracted from both living and dead molds. The PackageChina-Tibet goji fruits were preserved with sulphur dioxide which is a very effective fungicide. Therefore, no living molds were detected. No such information was given for Package-BIO-China goji fruits, but another processing could be used for these fruits, e.g., irradiation. However, only the bulk goji fruits were exposed to air while waiting for being sold. Thus, only the bulk goji fruits which were not packed in separate bags were contaminated by fungi according to the conventional method. On the other hand, fungi 

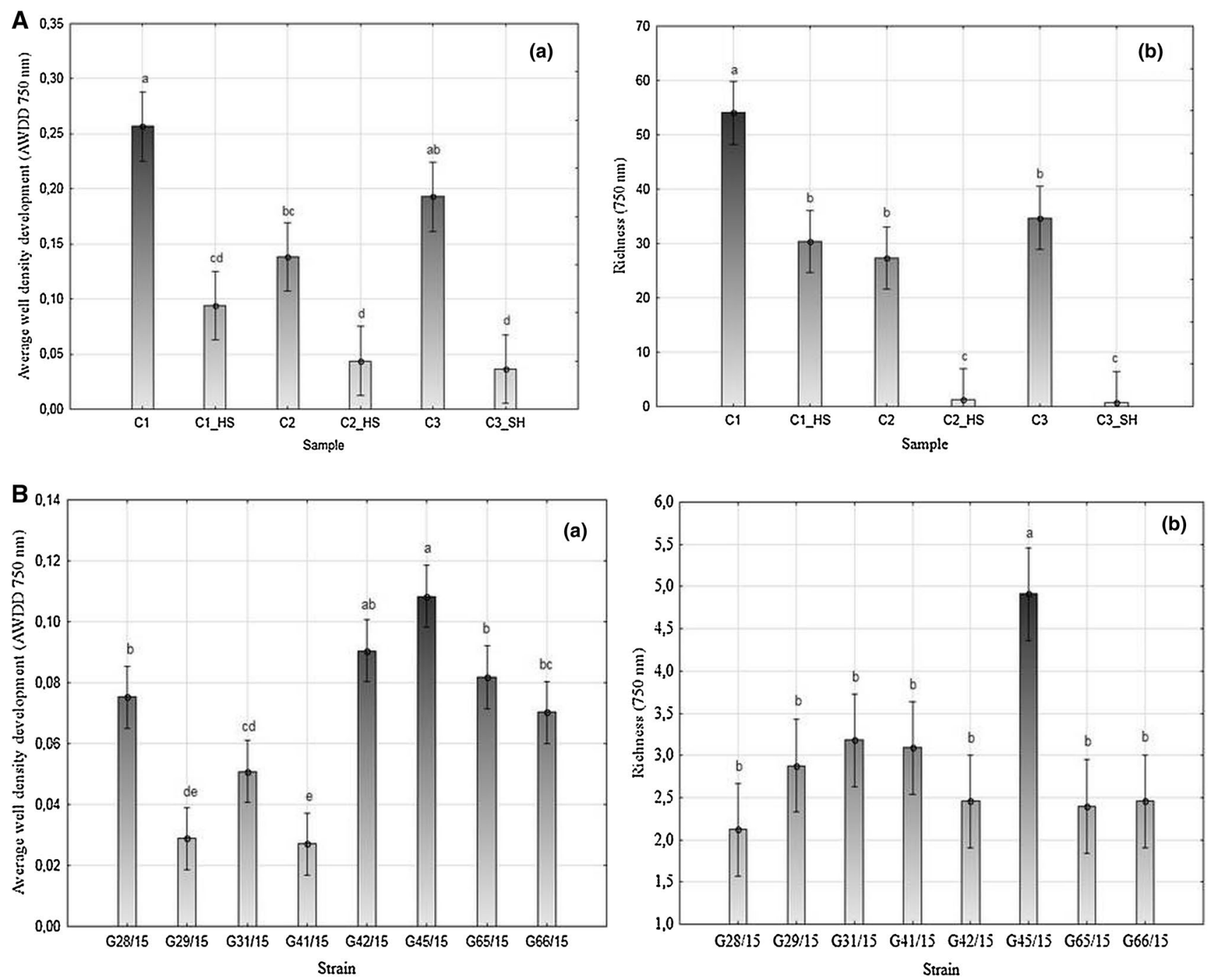

Fig. 3 Average well density development (AWDD) (Aa) and richness (Ab) of goji samples (A) and AWDD (Ba) and Richness (Bb) of strains of goji C1 sample (B). Vertical bars represent 0.95 confidence intervals. Averages with different letters are significantly different. Cl

were grown on all tested goji samples using the Biolog FF microplates, i.e., the community-level physiological profiles method. These results correlate with the ergosterol content.

It must be also mentioned that ergosterol content in sound and naturally contaminated grapes is on the same level as in other fruits $0.20-49.24 \mu \mathrm{g} \mathrm{g}^{-1}$, and it was found that the level of ergosterol below $0.5 \mu \mathrm{g} \mathrm{g}^{-1}$ is considered as minor ergosterol content [35]. Therefore, it might be confirmed that ergosterol content is low for Package-BIO-China $(0.36 \mu \mathrm{g}$ $\left.\mathrm{g}^{-1}\right)$ and Package-China-Tibet $\left(0.69 \mu \mathrm{g} \mathrm{g}^{-1}\right)$ and no fungal growth was found.

Besides, when fruits are contaminated by fungi, drying of fresh fruits at high temperature may induce heat-resistant fungi to form ascospores which can grow in appropriate conditions causing spoilage of drying fruits [20]. The results of

Bulk-China goji fruit, C1-HS Bulk-China goji fruit after heat shock, C2 Package-BIO-China, C2-HS Package-BIO-China after heat shock, $C 3$ Package-China-Tibet goji fruits, C3-HS Package-China-Tibet goji fruits after heat shock

dried goji fruits analysis showed contamination at the level of 28 and $23 \log _{10} \mathrm{CFU} \mathrm{g}^{-1}$ for fungi (yeast and molds) and molds, respectively. The previous research indicated fungal contamination of dried fruits such as apricots, cranberries, pineapple, and raisins; however, there are no data concerning goji fruit contamination by fungi [34]. The community-level physiological profile method was very useful for quick fungal detection in goji fruits, especially for heat-resistant fungi detection. The previous studies evaluating the occurrence of fungi in dried fruits showed that the main contamination of dried raisins, pineapple, apricots, and cranberries was molds such as Aspergillus sp. and Penicillium sp [34]. In contrary, the results of this study indicated that dried goji fruits were contaminated especially by heat-resistant yeast. According to our findings, raisins and cranberries fruits were not 


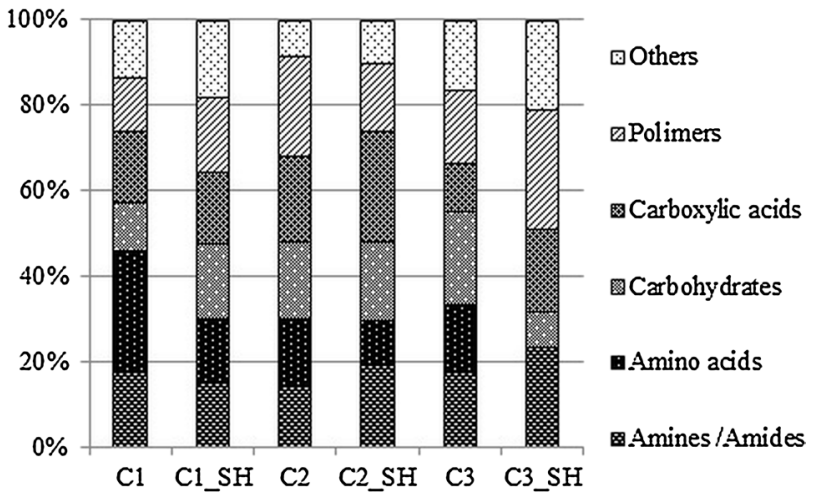

Fig. 4 C-substrate group utilization pattern of goji fruits fungal communities (Biolog ${ }^{\mathrm{TM}}$ ). C1 Bulk-China goji fruit, C1-HS Bulk-China goji fruit after heat shock, $C 2$ Package-BIO-China, $C 2-H S$ PackageBIO-China after heat shock, C3 Package-China-Tibet goji fruits, C3HS Package-China-Tibet goji fruits after heat shock

contaminated by fungi. The lack of fungal contamination can be a result of a postharvest microbe-destructing treatment. Imanaka et al. [36] also reported the absence of living molds in some dried fruits, while Tournas et al. [34] indicated that small percentage of the dried cranberry samples contained very low level of penicillia.

In this work, the potential of FF microplates (Biolog ${ }^{\mathrm{TM}}$ ) was addressed as a method for detection of fungi contamination, especially heat-resistant fungi contamination in dried fruits. The results indicated that catabolic activity of fungal communities is a good marker for the evaluation of mycological quality of dried fruits. Samples not contaminated by fungi did not show any signal expressed as substrate utilization, while, in contaminated fruits, substrates were utilized more efficiently. This effect was found as effective and accurate especially for the detection of heat-resistant fungi. Therefore, the approach for detection of this fungi group by FF microplates according to the developed procedure is noteworthy. The differences between the level of carbon substrate utilization in dried fruits and dried fruits treated by heat shock could be due to presence of heat-resistant ascospores in some fruit samples. To detect samples contaminated by these organisms, FF microplate method is useful and can prevent using of contaminated dried fruits and improve their quality.

\section{Conclusions}

Bioactive compounds in plant food diet such as phenolics and carotenoids from fruits are responsible for human health and can be used as markers in food assessment process. It was found that the dried goji fruits possessed higher content of total phenolics and antioxidant activity than the dried cranberries and raisins. Package condition of dried fruits had an influence on carotenoid content in goji fruits, but it had no influence on antioxidant activity, also in dried cranberries.

Quality of food depends not only on bioactive compounds but also on potential toxic substances and microorganisms including fungi. Ergosterol is produced by fungi; therefore, ergosterol content could be an indicator of fungal growth on dried fruits. Biolog FF plates and traditional culture methods were combined to study the fungal community as a fungal contamination marker of dried fruits. The methods were used not only for the detection of common fungi presence, but also for the detection of heat-resistant fungi. The differences in the number of fungi and heat-resistant fungi and ergosterol content led to fungal community variance on physiological level. Comparing the average well density development and richness of three samples of goji dried fruits, it was found that fungi in Bulk-China sample showed the highest diversity. In all tested samples, fungal communities showed tendency to metabolize substrates of amines/amides and carboxylic acids, which provides information deepening our understanding about fungal catabolic diversity of dried fruits.

The goji Bulk-China fruits were contaminated by heatresistant fungi; it could be reasoned that fungi in this sample after heat shock demonstrated higher substrate richness and the most intensive substrate utilization among the samples exposed to high temperature. Therefore, the Biolog FF microplates can be used according to developed procedure as quick, easy, cheap, and effective method for the detection of heat-resistant fungi in dried fruits.

Acknowledgements This work was supported by the 03/31/ DSMK/0345 Grant from the Polish Ministry of Science and Higher Education. The Biolog analyses were performed using equipment bought with European Union funds, The Eastern Poland Development Programme 2007-2013, and Regional Laboratory of Renewable Energy, IA PAS Grant no. POPW.01.03.00-06-005/09.

\section{Compliance with ethical standards}

Conflict of interest All authors declare that they have no conflict of interest.

Human and animal rights statement This article does not contain any studies with human or animal subjects.

Open Access This article is distributed under the terms of the Creative Commons Attribution 4.0 International License (http://creativeco mmons.org/licenses/by/4.0/), which permits unrestricted use, distribution, and reproduction in any medium, provided you give appropriate credit to the original author(s) and the source, provide a link to the Creative Commons license, and indicate if changes were made. 


\section{References}

1. Chang SK, Alasalvar C, Shahidi F (2016) Review of dried fruits: phytochemicals, antioxidant efficacies, and health benefits. J Funct Foods 21:113-132

2. Jeszka-Skowron M, Zgoła-Grześkowiak A, Stanisz E, Waśkiewicz A (2017) Potential health benefits and quality of dried fruits: goji fruits, cranberries and raisins. Food Chem 221:228-236

3. Anderson JA, Huth HA, Larson MM, Colby AJ, Krieg EJ, Golbach LP, Simon KA, Wasmundt SL, Malone CJ, Wilson T (2011) Glycaemic and insulin response to raisins, grapes and bananas in college aged students. FASEB J 25:587.4

4. Anderson JW, Waters AR (2013) Raisin consumption by humans: effects on glycemia and insulinemia and cardiovascular risk factors. J Food Sci 78:A11-A17

5. Karadeniz F, Durst RW, Wrolstad RE (2000) Polyphenolic composition of raisins. J Agric Food Chem 48:5343-5350

6. Ishiwata K, Yamaguchi T, Takamura H, Matoba T (2004) DPPH radical-scavenging activity and polyphenol content in dried fruits. Food Sci Technol Res 10:152-156

7. Joung KM, Jung HJ, Kim KN, Ho-Kyung K (2008) Effects of cranberry powder on serum lipid profiles and biomarkers of oxidative stress in rats fed an atherogenic diet. Nutr Res Pract 2(3):158-164

8. Oszmiański J, Kolniak-Ostek J, Lachowicz S, Gorzelany J, Matłok $\mathrm{N}$ (2015) Effect of dried powder preparation process on polyphenolic content and antioxidant capacity of cranberry (Vaccinium macrocarpon L.). Ind Crops Prod 77:658-665

9. Horbik D, Łowińska-Kluge A, Górski Z, Stanisz E, ZgołaGrześkowiak A (2013) Microwave-assisted extraction combined with HPLC-MS/MS for diagnosis of fungal contamination in building materials. J Braz Chem Soc 24:1478-1486

10. Saxena J, Munimbazi C, Bullerman LB (2001) Relationship of mould count, ergosterol and ochratoxin A production. Int J Food Microbiol 71:29-34

11. Stanisz E, Zgoła-Grześkowiak A, Waśkiewicz A, Stępień Ł, Beszterda M (2015) Can ergosterol be an indicator of fusarium fungi and mycotoxins in cereal products? J Braz Chem Soc 26:1-8

12. Makun H, Anjorin S, Moronfoye B, Adejo F, Afolabi O, Fagbayibo G, Balogun B, Surajudeen A (2010) Fungal and aliatoxin contamination of some human food commodities in Nigeria. Afr J Food Sci 4(4):127-135

13. Atanasova L, Druzhinina IS (2010) Review: global nutrient profiling by phenotype MicroArrays: a tool complementing genomic and proteomic studies in conidial fungi. J Zhejiang Univ Sci B 11(3):151-168

14. Feng S, Zhang H, Wang Y, Bai Z, Zhuang G (2009) Analysis of fungal community structure in the soil of Zoige Alpine Wetland. Acta Ecol Sin 29(5):260-266

15. Frąc M, Oszust K, Lipiec J, Jezierska-Tys S, Nwaichi EO (2014) Soil microbial functional and fungal diversity as influenced by municipal sewage sludge accumulation. Int J Environ Res Public Health 11(9):8891-8908

16. Janusz G, Czuryło A, Frąc M, Rola B, Sulej J, Pawlik A, Siwulski M, Rogalski J (2015) Laccase production and metabolic diversity among Flammulina velutipes strains. World J Microbiol Biotechnol 31:121-133

17. Frąc M, Oszust K, Lipiec J (2012) Community level physiological profiles (CLPP), characterization and microbial activity of soil amended with dairy sewage sludge. Sensors 12(3):3253-3268

18. Insam H, Goberna M (2004) Use of Biolog ${ }^{\circledR}$ for the community level physiological profiling (CLPP) of environmental samples.
In: Kowalchuk GA, de Bruijn F, Head IM, Van der Zijpp AJ, van Elsas JD (eds) Molecular microbial ecology manual. Springer, Netherlands, pp 853-860

19. Houbraken J, Samson R (2006) Standardization of methods for detecting heat resistant fungi. In: Hocking AD, Pitt JI, Samson RA, Thrane U (eds) Advances in experimental medicine and biology, vol 571. Springer, New York, pp 107-111

20. Frąc M, Jezierska-Tys S, Yaguchi T (2015) Occurrence, detection, and molecular and metabolic characterization of heat-resistant fungi in soils and plants and their risk to human health. Adv Agron 132:161-204

21. Scholte R, Samson R, Dijksterhuis J, Hoekstra E, Frisvad J (2004) Spoilage fungi in the industrial processing of food. In: Samson RA, Hoekstra ES, Frisvad JC (eds) Introduction to food-and airborne fungi, 7th edn. Netherlands, Utrecht, pp 339-356

22. Jeszka-Skowron M, Stanisz E, De Peña MP (2016) Relationship between antioxidant capacity, chlorogenic acids and elemental composition of green coffee. LWT Food Sci Technol 73:243-250

23. Cipolatti EP, Bulsing BA, De Sa CS, Burkert CAV, Furlong EB, De Medeiros Burkert JF (2015) Carotenoids from Phaffia rhodozyma: antioxidant activity and stability of extracts. Afr J Biotechnol 14(23):1982-1988

24. Rodriguez-Amaya DB (2001) A guide to carotenoid analysis in foods [online]. ILSI Press, Washington. ISBN: 1-57881-072-8. http://pdf.usaid.gov/pdf_docs/PNACQ929.pdf. Accessed 5 May 2018

25. Jeszka-Skowron M, Zgoła-Grześkowiak A (2014) Analysis of antioxidant activity, chlorogenic acid, and rutin content of Camellia sinensis infusions using response surface methodology optimization. Food Anal Methods 7:2033-2041

26. Amagase H, Farnsworth NR (2011) A review of botanical characteristics, phytochemistry, clinical relevance in efficacy and safety of Lycium barbarum fruit (Goji). Food Res Int 44:1702-1717

27. Endes Z, Uslu N, Özcan MM, Er F (2015) Physico-chemical properties, fatty acid composition and mineral contents of goji berry (Lycium barbarum L.) fruit. J Agroaliment Proc Technol 21(1):36-40

28. Peng Y, Ma C, Li Y, Leung KS, Jiang ZH, Zhao Z (2006) Quantification of zeaxanthin dipalmitate and total carotenoids in Lycium fruits. Plant Foods Hum Nutr 60:161-164

29. Namitha K, Negi PS (2010) Chemistry and biotechnology of carotenoids. Crit Rev Food Sci Nutr 50:728-760

30. Vinson JA, Zubik L, Bose P, Samman N, Proch J (2005) Dried fruits: excellent in vitro and in vivo antioxidants. J Am Coll Nutr 24:44-50

31. Luttfullah G, Hussain A (2011) Studies on contamination level of aflatoxins in some dried fruits and nuts of Pakistan. Food Control $22: 426-429$

32. Masood M, Iqbal SZ, Asi MR, Malik N (2015) Natural occurrence of aflatoxins in dry fruits and edible nuts. Food Control 55:62-65

33. Park JN, Sung NY, Byun EH, Byun EB, Song BS, Kim YH, Lee KA, Son EJ, Lyu ES (2015) Microbial analysis and survey test of gamma-irradiated freeze-dried fruits for patient's food. Radiat Phys Chem 111:57-61

34. Tournas VH, Niazi NS, Kohn JS (2015) Fungal presence in selected tree nuts and dried fruits. Microbiol Insight 8:1-6

35. Porep JU, Walter R, Kortekamp A, Carle R (2014) Ergosterol as an objective indicator for grape rot and fungal biomass in grapes. Food Control 37:77-84

36. Imanaka BT, Taniwaki MH, Menezes HC, Vicente E, Fungaro MH (2005) Incidence of toxigenic fungi and ochratoxin A in dried fruits sold in Brazil. Food Addit Contam 22:1258-1263 